\title{
Polymerase Chain Reaction Versus Blood Culture to Detect Candida Species in High-Risk Patients with Suspected Invasive Candidiasis: The MICAFEM Study
}

\author{
Mercedes Nieto - Juan Carlos Robles - Manuel Causse · \\ Leticia Gutiérrez • Maria Cruz Perez • Ricard Ferrer • Mariona Xercavins · \\ Eugenio Herrero $\cdot$ Elia Sirvent $\cdot$ Cristina Fernández $\cdot$ Paloma Anguita $\cdot$ \\ Paloma Merino on behalf of the MICAFEM Study Group, GEIPC (SEIMC) and GTEIS (SEMICYUC)
}

Received: February 11, 2019 / Published online: May 24, 2019

(c) The Author(s) 2019

\section{ABSTRACT}

Introduction: We evaluated the diagnostic reliability of serum polymerase chain reaction (PCR) versus blood culture, abdominal fluid or both (composite measure) in patients receiving empirical antifungal treatment for suspected invasive candidiasis.

The members of the MICAFEM Study Group, GEIPC (SEIMC) and GTEIS (SEMICYUC) are listed in Acknowledgements.

Enhanced Digital Features To view enhanced digital features for this article go to https://doi.org/10.6084/ m9.figshare.8124113.

Electronic supplementary material The online version of this article (https://doi.org/10.1007/s40121019-0248-z) contains supplementary material, which is available to authorized users.

M. Nieto $(\bowtie) \cdot$ C. Fernández · P. Merino Hospital Clínico San Carlos, Madrid, Spain e-mail: mnietocabrera@hotmail.com

J. C. Robles · M. Causse

Hospital Reina Sofía, Córdoba, Spain

L. Gutiérrez · M. Cruz Perez

Hospital Universitario Son Llatzer, Palma de Mallorca, Spain

R. Ferrer · M. Xercavins

Catlab-Hospital Universitari Mutua Terrassa,

Barcelona, Spain
Methods: This observational, prospective, noninterventional, multicentre study in Spain enrolled 176 critically ill patients admitted to the intensive care unit. Separate blood samples for culture and serum PCR were taken before the start of antifungal therapy. Patient assessment was performed according to each site's usual clinical practice. The primary end point was concordance between serum PCR and blood culture. Secondary end points were concordance between serum PCR and a positive abdominal fluid sample or the composite measure. Quality indices included sensitivity, specificity, positive/negative predictive values (PPV/NPV) and kappa indices.

Results: Among 175 evaluable patients, rates of Candida detection were similar for serum PCR $(n=16 / 175,9.1 \%)$ versus blood culture $(n=14$ / $175,8.0 \%)$. Quality indices for serum PCR relative to blood culture were: sensitivity $21.4 \%$;

\section{R. Ferrer}

Hospital Vall d’Hebron, Barcelona, Spain

E. Herrero · E. Sirvent

Hospital Universitario de Torrevieja, Alicante, Spain

P. Anguita

Astellas Pharma Inc., Madrid, Spain 
specificity 91.9\%; PPV 18.8\%; NPV 93.1\%; kappa index 0.125. Thirty-two abdominal fluid samples were positive. Quality indices for serum PCR versus abdominal fluid were: sensitivity 31.3\%; specificity 83.0\%; PPV 15.6\%; NPV 92.3\%; kappa index 0.100. Quality indices for serum PCR versus the composite measure were: sensitivity $15.8 \%$; specificity $92.7 \%$; PPV $37.5 \%$; NPV 79.9\%; kappa index 0.107.

Conclusion: The sensitivity of serum PCR for Candida detection was low and the rate of concordance was low between serum PCR and the other diagnostic techniques used to identify Candida infections. Hospital-based diagnostic tests need optimising to improve outcomes in patients with suspected invasive candidiasis.

Funding: Astellas Pharma Inc.

Keywords: Abdominal fluid; Blood cultures; Candida; Candida diagnosis; Empirical antifungal treatment; Invasive candidiasis; Intensive care unit; PCR

\section{INTRODUCTION}

Candida species are among the top ten bloodstream pathogens [1] and remain the most common cause of invasive fungal infections worldwide [2-5]. There is wide inter-regional variability in the incidence of blood infections due to Candida spp. (candidaemia). One review suggests the incidence may vary between 1.2 and 25 cases per 100,000 inhabitants [6]. Invasive candidiasis, which occurs when Candida spp. infects other tissues and organs, causes significant morbidity and high mortality rates [1, 7-9]. Furthermore, the costs associated with invasive candidiasis are substantial because of the need to use additional diagnostic procedures, prolonged hospitalisation and extra medications [10-12].

Patients with haematological malignancies or neutropenia, those undergoing gastrointestinal surgery, premature infants or patients aged $>70$ years are at increased risk for acquiring candidaemia during hospitalisation due to an underlying medical condition [13]. Within these high-risk groups, specific factors further increase the risk for invasive candidiasis, for instance, surgery, the presence of vascular catheters, mucosal colonisation with Candida spp., sepsis, length of previous antibiotic therapy and receipt of total parenteral nutrition [13-20]. These risk factors are frequently encountered in critically ill patients in the intensive care unit (ICU) [21] where the incidence of candidaemia is tenfold higher compared with other hospital wards, and mortality rates are between 25 and 50\% [22-25].

The difficulties in obtaining an early and accurate diagnosis of invasive candidiasis in critically ill patients have led to the widespread use of empirical antifungal treatment in such patients [25-27]. Empirical antifungal treatment in high-risk patients is currently recommended by the European Society for Clinical Microbiology and Infectious Diseases [28] as well as by the Infectious Diseases Society of America [29]. There is moderate-quality evidence showing that empirical antifungal therapy with an echinocandin can be effective in high-risk settings; therefore, the echinocandins are strongly recommended for empiric treatment in non-neutropenic patients with suspected invasive candidiasis within an ICU setting [30]. However, this strategy may contribute to the overuse of antifungals and could increase the risk of antifungal resistance, which may have implications for the length of hospital stay, lead to adverse patient outcomes and potentially increase healthcare costs [9, 31, 32].

Blood culture is the current 'gold' standard for diagnosing invasive candidiasis, despite its numerous limitations, which include delays of up to $48 \mathrm{~h}$ until results are available for the majority of patients $[33,34]$ and possibly longer when information on specific species is required [35]. As it has been shown that delays in initiating appropriate treatment significantly increase the all-cause mortality rate, independently of other risk factors [33, 34], a prompt and accurate diagnosis of invasive candidiasis is crucially important. In addition, blood cultures show positivity in only a minority of cases (especially in patients with deep-seated candidiasis) and often only late during the course of infection [1].

To overcome these difficulties, polymerase chain reaction (PCR)-based assays have been 
introduced as an alternative tool for diagnosing invasive candidiasis [25]. The advantages of PCR testing include same-day identification of Candida spp. and the potential for relatively fast and continuous monitoring of persistence or resolution of infection [36]. A comprehensive systematic review and meta-analysis of PCR diagnosis for invasive candidiasis has been conducted: 54 studies comprising 4694 patients (of whom 963 had proven, probable or possible invasive candidiasis) were included. The results from this meta-analysis demonstrated that PCR can have a higher diagnostic sensitivity than blood culture, with pooled sensitivity and specificity values of $95 \%$ and $92 \%$, respectively. Importantly, PCR allowed the earlier diagnosis of Candida infections [36].

The aims of the current study were to evaluate the diagnostic reliability of serum PCR versus either blood culture or abdominal fluid samples (and both as a composite measure) and to assess the clinical response to antifungal therapy in critically ill patients with suspected invasive candidiasis admitted to ICUs across Spain.

\section{METHODS}

\section{Study Design and Population}

This was a prospective, multicentre, observational, non-interventional study (Astellas study no. AST-INF-2012-01) conducted in 27 centres and 9 regions across Spain. The date of first enrolment was 18 March 2013 and the last evaluation was completed on 2 January 2015. An Independent Ethics Committee or an Institutional Review Board reviewed and approved the protocol, protocol amendments and patient information before the start of the study. The "master" ethics committee was the H. Clinico San Carlos committee (a list of all ethics approval bodies that approved the study in the various centres is included in the supplementary material). The study was conducted in accordance with the principles of the 1964 Declaration of Helsinki and its later amendments, Good Clinical Practice guidelines and International Conference on Harmonisation guidelines. Informed consent was provided by all patients, or their legal representative, before the start of the study.

Inclusion criteria were male or female patients aged $\geq 18$ years admitted to the ICU and requiring empirical antifungal therapy because of a high risk of invasive candidiasis. Patients meeting at least one of the following criteria were considered at high risk: those with a Candida score $\geq 3$ (based on severe sepsis, 2 points; surgery, 1 point; parenteral nutrition, 1 point; Candida at multiple sites of infection, 1 point); patients with sepsis and $\geq 3$ risk factors for invasive candidiasis (from severe pancreatitis, extra-renal depuration, broad-spectrum antibiotic treatment for $>7$ days, abdominal surgery, ICU stay of $>15$ days, parenteral nutrition, central venous catheterisation, Candida at multiple sites of infection, candiduria and Acute Physiology and Chronic Health Evaluation score $>15$ ); or immunocompromised patients with sepsis. Patients with documented candidiasis at enrolment, patients taking antifungals at the time of screening or those with any condition that, in the opinion of the investigator, rendered their participation inadvisable were excluded from enrolment.

\section{Treatment and Response Criteria}

This was a non-interventional study. Patients were recruited consecutively and managed and treated according to the physicians' usual clinical practice. The decision to prescribe antifungal therapy was made prior to (and independently from) the patient's inclusion in the study. Once the decision had been made to initiate treatment, patients were recruited into the study and the pertinent data and samples were collected at enrolment prior to initiating antifungal treatment, which was then administered according to the physicians' usual practice.

Any other treatments that the patients could receive were according to the treating physician's regular clinical practice and each site's health care protocols.

Investigators were asked to make a clinical assessment of the clinical and microbiological 


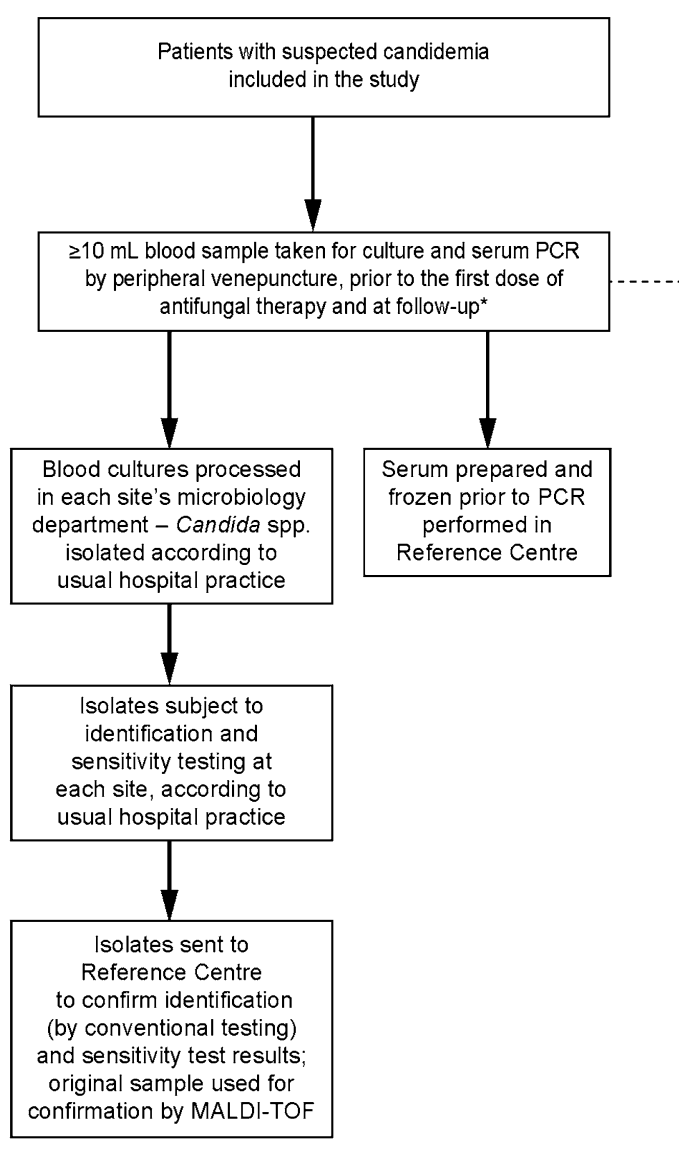

Fig. 1 Microbiological analyses. ${ }^{*}$ Patients meeting the following criteria were considered at high risk: those with a Candida score $\geq 3$ (based on severe sepsis, 2 points; surgery, 1 point; parenteral nutrition, 1 point; Candida at multiple sites of infection, 1 point); patients with sepsis and $\geq 3$ risk factors for invasive candidiasis (from severe acute pancreatitis, extra-renal depuration, broad-spectrum antibiotic treatment for $>7$ days, major abdominal surgery, ICU stay of $>15$ days, parenteral nutrition, central venous catheterisation, Candida at multiple sites of infection, candiduria and Acute Physiology and Chronic Health Evaluation score $>15$ ); or sepsis in

response to empirical antifungal treatment for the suspected invasive candidiasis.

\section{Microbiological Analyses}

Microbiological analyses are summarised in Fig. 1. A blood sample ( $\geq 10 \mathrm{ml}$ ) for culture and serum PCR was taken by peripheral venepuncture, before the first dose of antifungal therapy.

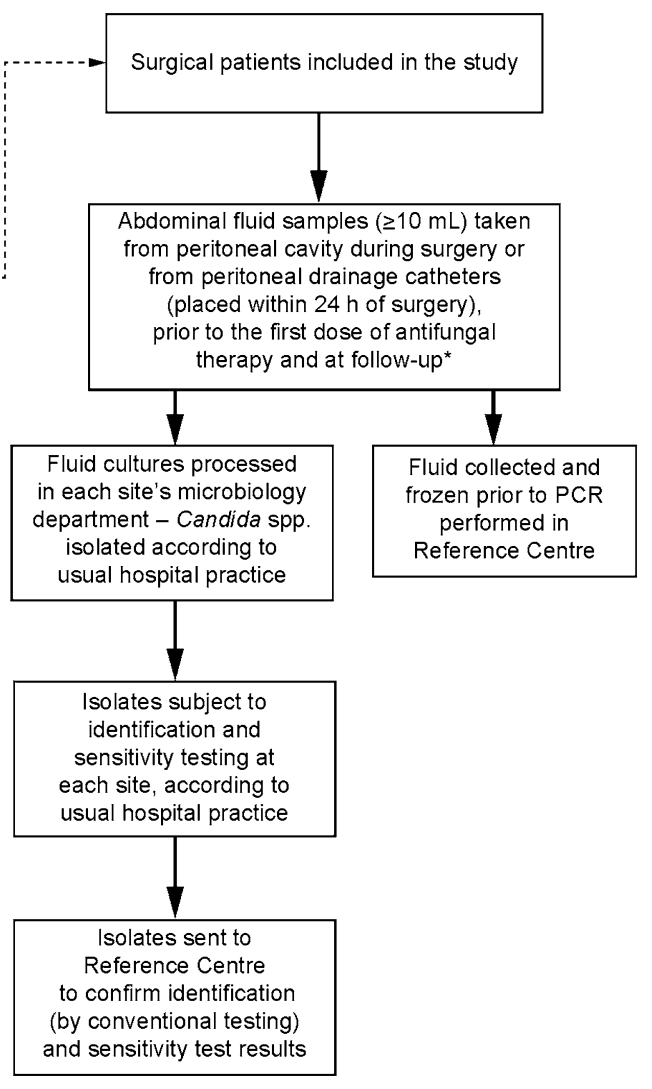

immunocompromised patients with neutropenia, haematological disease, solid organ or bone marrow transplantation, treatment with immunosuppressive drugs, chemotherapy or monoclonal antibodies; or human immunodeficiency virus infection. ${ }^{* *}$ Blood sample times were as follows: at baseline (before initiating antifungal treatment) and at follow-up (blood cultures were taken according to the site's usual clinical practice). Serum PCR was performed at the same time as the blood culture sample. ICU intensive care unit, MALDI-TOF matrixassisted laser desorption/ionisation time of flight, $P C R$ polymerase chain reaction

Follow-up blood samples for culture and serum PCR were taken according to the site's usual clinical practice/guideline recommendations 3 and 7 days after the baseline sample. Blood sample collection and management were standardised across the study centres. Samples for PCR were sent to the National Microbiology Centre (CNM).

Blood cultures were processed within each site's microbiology department, according to 
usual hospital practice, with identification of isolated strains. When a Candida spp. was isolated, identification and sensitivity tests were performed within each site, according to a standardised protocol. The original blood samples were then frozen and sent to the Reference Centre (National Microbiology Centre, Hospital Clínico San Carlos) to confirm the initial identification and sensitivity results. At the Reference Centre, Candida isolates were identified, and the sensitivity results determined by the minimum inhibitory concentration to antifungal therapy were confirmed by conventional testing, with the original sample used to confirm identification by matrix-assisted laser desorption/ionisation time-of-flight mass spectrometry.

The remainder of the blood sample used for the PCR assay was centrifuged at $3000 \mathrm{rpm}$ for $10 \mathrm{~min}$ to separate the serum, which was then frozen at $-20^{\circ} \mathrm{C}$. The frozen serum samples were stored at each site's microbiology department until they were sent to the Reference Centre. All the samples were identified by specifically designed labels, and the sites also had specially designed data sheets for accurate sample matching and identification.

Abdominal fluid samples were obtained from the peritoneal cavity during laparotomy or from peritoneal drainage catheters (placed within $24 \mathrm{~h}$ of surgery) and were cultured according to each site's regular clinical practice and subjected to sensitivity testing. When Candida spp. were identified, isolates were sent to the Reference Centre to confirm the initial identification and sensitivity results by conventional testing.

\section{Multiplex Real-Time PCR Assay}

Deoxyribonucleic acid (DNA) for PCR assays was extracted from blood and sera using the QIAamp $^{\circledR}$ DNA Mini kit (Qiagen, Izasa, Madrid, Spain), following the manufacturer's instructions. DNA was eluted in $50 \mu \mathrm{l}$ of buffer, and $2.2 \mu \mathrm{l}$ of DNA extracted from each sample was used for subsequent PCR. Two multiplex PCR assays, developed by Fortun et al. (2014), were used to detect six Candida species common in IC (Candida albicans, C. parapsilosis, C. tropicalis,
C. glabrata, C. krusei, and C. guilliermondii); these PCR assays have been described in detail elsewhere [25]. Briefly, Candida spp. were detected using molecular probes with fluorescent dyes (FAM, HEX, ROX, and CYAN 500). Probes and primers were designed based on the ITS1/2 sequences of ribosomal DNA regions of Candida strains belonging to the Spanish National Center of Microbiology collection, using Beacon Designer 5.0 software (Premier Biosoft, Palo Alto, CA, USA). Two multiplex PCRs were performed simultaneously in the LightCycler 480 system (Roche Diagnostics, Mannheim, Germany), one using the LightCycler Probes Master Kit (Roche Diagnostic, Madrid, Spain) to detect C. albicans, C. parapsilosis and C. tropicalis and one using the $2 \times$ Sensimix Probe Kit (Quantace, Ecogen, Madrid, Spain) to detect C. glabrata, C. krusei and C. guilliermondii. Samples were analysed in duplicate and standards were run with each set of samples and negative controls. The following CT values were used to define a positive PCR result: C. albicans (20 fg DNA) 34.15-37.44; C. parapsilosis (20 fg DNA) $31.45-35.39 ; \quad$ C. tropicalis (20 fg DNA) 32.34-36.16; C. glabrata (200 fg DNA) 31.10-36.24; C. krusei (20 fg DNA) 34-38 (200 fg DNA) 30.80-33.80; C. guilliermondii (20 fg DNA) 38.40-42.31. The overall result was considered positive if both PCRs for each sample were positive according to the laboratory standard. Sensitivity was determined at the Reference Centre; isolates were identified and the sensitivity results, determined by the minimum inhibitory concentration of Candida isolates to antifungal therapy, were confirmed by conventional testing.

Those who conducted the blood/abdominal fluid tests were blinded to the results of the PCR analyses, and vice versa.

A diagnosis of invasive candidiasis was made when a positive blood culture or positive abdominal fluid sample was obtained from a surgical setting or a peritoneal drainage catheter (placed within $24 \mathrm{~h}$ of surgery) or when a positive PCR was recorded in samples from patients whose clinical condition improved with antifungal treatment. 


\section{End Points}

The primary end point was the degree of concordance between the results of the serum PCR and blood culture. Secondary end points were the degree of concordance between the results for serum PCR and a positive abdominal fluid culture and positivity for blood culture or abdominal fluid culture (assessed as a composite measure).

The relationship between the presence of invasive candidiasis and the risk factors that contribute to a patient's high-risk status (Candida score $\geq 3$; sepsis and $\geq 3$ risk factors for invasive candidiasis; sepsis and immunosuppression) was also investigated.

Data were collected from all patients at the time of enrolment and, if available, when follow-up blood cultures were performed, on discharge from the ICU and at the end of antifungal treatment.

\section{Statistical Analysis}

The sample size was calculated based on the primary end point using estimates of the sensitivity of serum PCR (80\%) and blood culture $(50 \%)$, based on the published literature $[35,37]$, and the kappa index of concordance between both techniques $(0.3)$ in patients with suspected invasive candidiasis, admitted to ICUs in which empirical treatment was initiated with an antifungal. Taking these estimates into account, a sample size of 179 patients would provide precision of \pm 0.1 to estimate concordance between serum PCR and blood culture diagnoses, with 95\% confidence intervals (CIs). Using the assumption that $10 \%$ of patients would not be eligible, the planned sample size was 199 . The sample size calculation was performed using EpiData software, version 3.1 (The EpiData Association, Odense, Denmark).

The analysis population included all patients who fulfilled the entry criteria and were considered evaluable. Concordance between the results of serum PCR and blood culture (primary end point) was assessed by estimating the kappa index. The kappa index, which assesses intertest reliability, can range from -1 to +1 , with higher scores indicating greater concordance [38].

The sensitivity, specificity, positive predictive value (PPV) and negative predictive value (NPV) and the respective 95\% CIs were calculated for serum PCR relative to blood culture.

Positive and negative odds ratios (ORs) and post-test probabilities [positive post-test probability (PPTP) and negative post-test probability (NPTP)] were also determined.

Concordance between the results of serum PCR versus abdominal fluid culture and versus the composite measure were analysed in the same way as the primary end point. The relationship between the presence of invasive candidiasis and patient risk factors (Candida score $\geq 3$; sepsis and $\geq 3$ risk factors for invasive candidiasis; sepsis and immune suppression) was analysed using a Chi-squared test. Binary logistic regression models were used to determine which risk factors had the greatest weight, according to the microbiological test used. These models were executed hierarchically and with the stepwise backward method. Data were presented as ORs and 95\% CIs.

An adjusted binary logistic regression model (with a bilateral significance level of 0.10 , Chisquared test) was used to evaluate which of the individual risk factors were related to the presence of invasive candidiasis according to blood culture results. All calculations were performed with STATA for Windows, version 12 (http:// www.stata.com/).

\section{RESULTS}

\section{Patient Population}

Overall, 176 patients were recruited into the study; of these, 175 patients were regarded as evaluable and included in the analysis set.

Of the total evaluable patients, 125 (71.4\%) completed the study period and 50 (28.6\%) withdrew early. Among the patients who withdrew early, 37 died (21.1\% of the total evaluable patients). The most common reasons for death were multi-organ failure and septic shock [ $n=17(9.7 \%)$ and $n=10$ (5.7\%), respectively]. 


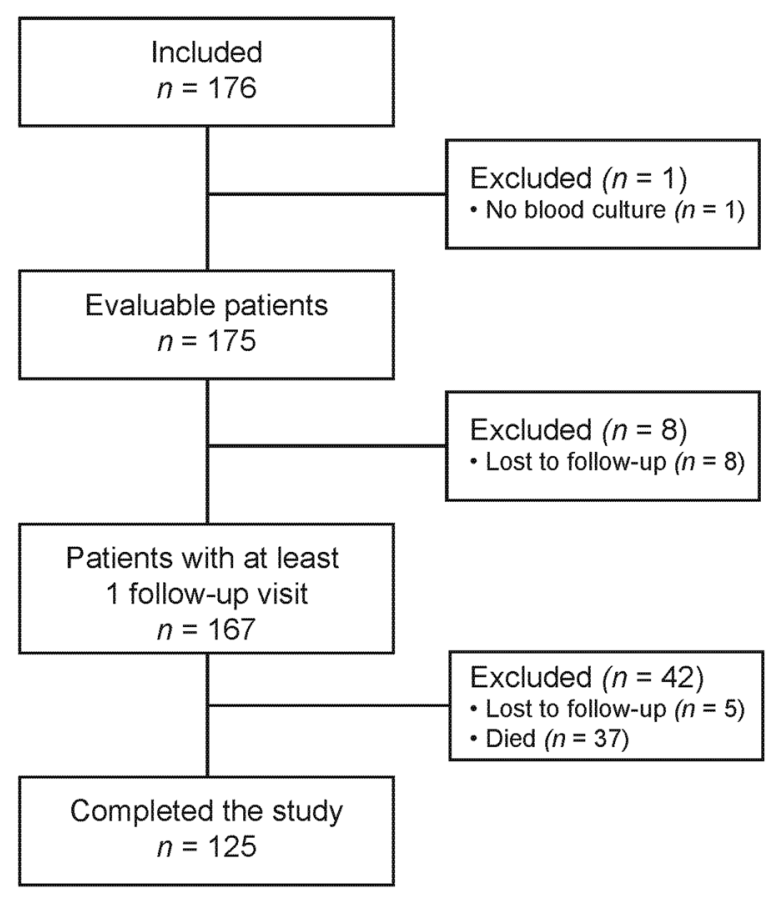

Fig. 2 Flow chart of the study population

A flow diagram detailing patient disposition is presented in Fig. 2.

Demographic data and clinical characteristics are summarised in Table 1 . The mean age was 67 years and most patients were male. The mean duration of stay in the ICU was 6.3 days. A total of $132(75 \%)$ patients had a Candida score $\geq 3$ and 169 (96.0\%) had sepsis. In total, 99 patients $(56.3 \%)$ had concomitant infections and 167 (94.9\%) were receiving concomitant antibiotic treatment. The median number of days that patients were in the ICU before initiation of antifungal treatment was 5 days (interquartile range, 1-12 days).

\section{Empirical Antifungal Treatment}

In patients with available data $(n=116)$, the mean (SD) duration of antifungal treatment was 21.72 (17.50) days. For participants who received $\geq 1$ follow-up visit $(n=167)$, the microbiological response was non-evaluable for the majority of patients $(n=122 / 167,73.1 \%)$. According to clinical criteria, $34(20.4 \%)$ of patients were cured, 76 (45.5\%) were improved and $57(34.1 \%)$ experienced treatment failure.
Candida eradication was achieved in $28 / 167$ (16.8\%) patients, but persisted (at the end of antifungal treatment) in $17 / 167 \quad(10.2 \%)$ patients.

\section{Primary End Point}

Rates of detection of Candida infection were similar for blood culture and serum PCR $[n=14 / 175 \quad(8.0 \%)$ with blood culture and $n=16 / 175$ (9.1\%) with serum PCR]. C. albicans was the most common pathogen and was detected with a greater frequency with serum PCR $(14 / 16,87.5 \%)$ than with blood culture (8/ $14,57.1 \%)$. Other pathogens that were detected using both techniques were C. glabrata $(12.5 \%$ and $42.9 \%$, respectively) and C. krusei $(12.5 \%$ and $7.1 \%$, respectively). C. tropicalis and $C$. parasilopsis were detected in one sample each using blood culture.

Concordance rates and quality indices for the results of blood culture versus serum PCR are shown in Table 2 . The sensitivity of serum PCR relative to blood culture was $21.4 \%$ and the specificity was $91.9 \%$; the PPV was $18.8 \%$ and the NPV was $93.1 \%$, while the PPTP and NPTP were $18.8 \%$ and $6.9 \%$, respectively, indicating that the concordance of serum PCR using blood culture as a reference was very low, as confirmed by the kappa value (0.125) (Table 2$)$.

\section{Secondary End Points}

Thirty-two patients had positive results for Candida spp. in the abdominal fluid. The most common species were $C$. albicans $[n=18$ $(56.3 \%)]$ and C. glabrata $[n=9(28.1 \%)]$.

Concordance rates and quality indices for the results for serum PCR versus abdominal fluid samples are also shown in Table 2. The sensitivity and specificity of serum PCR relative to abdominal fluid were $31.3 \%$ and $83.0 \%$, respectively. The PPV and NPV were $15.6 \%$ and 92.3\%, respectively; PPTP was $17.0 \%$ and NPTP was $7.7 \%$. The kappa index was 0.100 , also indicating a low rate of concordance between serum PCR and abdominal fluid samples.

When the results of serum PCR versus the composite measure of blood culture plus 
Table 1 Demographics and clinical characteristics at baseline

\begin{tabular}{|c|c|}
\hline Patient characteristic & $\begin{array}{l}\text { Mean (SD), } \\
\text { unless } \\
\text { specified } \\
(N=176)\end{array}$ \\
\hline \multicolumn{2}{|l|}{ Gender, $n(\%)$} \\
\hline Male & $115(65.3)$ \\
\hline Female & $61(34.7)$ \\
\hline Age (years) & $66.6(12.7)$ \\
\hline BMI $\left(\mathrm{kg} / \mathrm{m}^{2}\right)$ & $28.0(6.4)$ \\
\hline \multicolumn{2}{|l|}{ Reason for ICU admission, $n$ (\%) } \\
\hline Medical & $88(50.0)$ \\
\hline Surgical & $88(50.0)$ \\
\hline Abdominal & $81(46.0)$ \\
\hline Chest & $4(2.3)$ \\
\hline Cardiovascular & $4(2.3)$ \\
\hline Orthopaedic and trauma surgery & $1(0.6)$ \\
\hline Other & $2(1.1)$ \\
\hline Duration of ICU stay (days) & $6.3(9.9)$ \\
\hline Candida score & $3.10(1.16)$ \\
\hline \multicolumn{2}{|l|}{ Patients with Candida score, $n$ (\%) } \\
\hline$<3$ & $44(25.0)$ \\
\hline 3 & $56(31.8)$ \\
\hline 4 & $67(38.1)$ \\
\hline 5 & $9(5.1)$ \\
\hline Patients with sepsis, $n$ (\%) & $169(96.0)$ \\
\hline Severe & $43(24.4)$ \\
\hline Septic shock & $103(58.5)$ \\
\hline \multicolumn{2}{|l|}{ Patients considered at high risk, $n(\%)$} \\
\hline Candida score $\geq 3$ & $132(75.0)$ \\
\hline Serious sepsis ( 2 points) & $147(83.5)$ \\
\hline Prior abdominal surgery ( 1 point) & $116(65.9)$ \\
\hline Parenteral nutrition ( 1 point) & $108(61.4)$ \\
\hline Multiple colonisation ( 1 point) & $27(15.3)$ \\
\hline
\end{tabular}

Table 1 continued

\begin{tabular}{|c|c|}
\hline Patient characteristic & $\begin{array}{l}\text { Mean }(\mathrm{SD}) \text {, } \\
\text { unless } \\
\text { specified } \\
(N=176)\end{array}$ \\
\hline Sepsis in patients with $\geq 3$ risk factors & $163(92.6)$ \\
\hline Serious acute pancreatitis & $24(13.6)$ \\
\hline Extrarenal depuration & $56(31.8)$ \\
\hline Broad-spectrum antibiotics $>7$ days & $105(59.7)$ \\
\hline Major abdominal surgery & $116(65.9)$ \\
\hline Admission to $\mathrm{ICU}>15$ days & $51(29.0)$ \\
\hline Parenteral nutrition & $109(61.9)$ \\
\hline Central venous catheter & $162(92.0)$ \\
\hline Colonisation in 2 or more locations & $27(15.3)$ \\
\hline Candiduria & $21(11.9)$ \\
\hline APACHE on admission $>15$ & $120(68.2)$ \\
\hline Diabetes mellitus & $39(22.2)$ \\
\hline Major burns (serious burns) & $0(0)$ \\
\hline $\begin{array}{l}\text { Sepsis in patients with } \\
\text { immunosuppression }\end{array}$ & $36(20.5)$ \\
\hline Neutropenia & $5(2.8)$ \\
\hline Haematological disease & $4(2.3)$ \\
\hline $\begin{array}{l}\text { Solid organ or bone marrow } \\
\text { transplantation }\end{array}$ & $9(5.1)$ \\
\hline $\begin{array}{l}\text { Treatment with immunosuppresive } \\
\text { drugs, chemotherapy agents or } \\
\text { monoclonal antibodies }\end{array}$ & $29(16.5)$ \\
\hline Human immunodeficiency virus & $5(2.8)$ \\
\hline
\end{tabular}

$B M I$ body mass index, $I C U$ intensive care unit, $S D$ standard deviation

abdominal fluid were examined, the quality indices remained comparable (sensitivity 15.8\%; specificity 92.7\%; PPV 37.5\%; NPV $79.9 \%$ ). The kappa index was 0.107 , again indicating a very low rate of concordance between serum PCR and the composite measure. 
Table 2 Quality indices for serum PCR, blood culture, abdominal fluid and the composite measure in patients with suspected invasive candidiasis

\begin{tabular}{llll}
\hline Parameter & $\begin{array}{l}\text { PCR vs. blood } \\
\text { culture }(\boldsymbol{n}=\mathbf{1 7 5})\end{array}$ & $\begin{array}{l}\text { PCR vs. abdominal } \\
\text { fluid }(\boldsymbol{n}=\mathbf{1 7 5})\end{array}$ & $\begin{array}{l}\text { PCR vs. composite measure } \\
\text { (blood culture and } \\
\text { abdominal fluid) } \boldsymbol{n}=\mathbf{1 7 5})\end{array}$ \\
\hline True positive, $n$ & 3 & 5 & 6 \\
False negative, $n$ & 11 & 27 & 32 \\
False positive, $n$ & 13 & 11 & 10 \\
True negative, $n$ & 148 & 132 & 127 \\
Sensitivity, \% (95\% CI) & $21.4(0.0-42.9)$ & $31.3(8.5-54.0)$ & $15.8(4.2-27.4)$ \\
Specificity, \% (95\% CI) & $91.9(87.7-96.1)$ & $83.0(77.2-88.9)$ & $92.7(88.3-97.1)$ \\
PPV, \% (95\% CI) & $18.8(0.0-37.9)$ & $15.6(3.0-28.2)$ & $37.5(13.8-61.2)$ \\
NPV, \% (95\% CI) & $93.1(89.1-97.0)$ & $92.3(87.9-96.7)$ & $79.9(73.6-86.1)$ \\
PLR, ratio (95\% CI) & $2.65(0.86-8.22)$ & $2.03(0.37-2.68)$ & $2.16(0.84-5.57)$ \\
NLR, ratio (95\% CI) & $0.85(0.63-1.17)$ & $0.91(0.88-1.13)$ & $0.91(0.75-1.10)$ \\
PPTP, \% (95\% CI) & $18.8(0.0-37.9)$ & $17.0(4.0-20.0)$ & $37.5(13.8-61.2)$ \\
NPTP, \% (95\% CI) & $6.9(3.0-10.9)$ & $7.7(3.3-10.0)$ & $20.1(13.9-26.3)$ \\
Kappa index (95\% CI) & $0.125(0.00-0.33)$ & $0.100(0.00-0.26)$ & $0.107(0.00-0.26)$ \\
\hline
\end{tabular}

$C I$ confidence interval, $N L R$ negative likelihood ratio, $N P T P$ negative post-test probability, $N P V$ negative predictive value, $P C R$ polymerase chain reaction, $P L R$ positive likelihood ratio, $P P T P$ positive post-test probability, $P P V$ positive predictive value

\section{Risk Factors for Invasive Candidiasis}

Results of the analysis of the relationship between the presence of invasive candidiasis and patient risk factors are summarised in Table 3 . The only significant relationships were between the presence of invasive candidiasis as determined using abdominal fluid/the composite measure and a candidiasis score $\geq 3$ (both $P=0.006)$.

According to the logistic regression analyses (Table 4), patients with a Candida score $\geq 3$ were seven times more likely to present with invasive candidiasis than those with a lower Candida score when assessed by blood culture, but this result was non-significant $(P=0.100)$. They were also 4.15 times more likely present with invasive candidiasis when assessed using the composite measure $(P=0.030)$. When assessed using serum PCR, none of the three risk factors influenced the probability of being positive for Candida spp. (Table 4).

Patient risk factors that were positively but not statistically significantly associated with the presence of invasive candidiasis according to blood culture results, using the adjusted binary logistic regression model, were abdominal surgery (OR 4.342; $P=0.068)$ and an ICU stay $>15$ days (OR 2.560, $P=0.090$ ) (Table 5). There was also a $44 \%$ relative reduction in the risk of invasive candidiasis in non-immunosuppressed versus immunosuppressed patients (OR 0.556), but this was not statistically significant $(P=0.419)$ (Table 5).

\section{DISCUSSION}

The primary objective of this study was to examine the concordance between the results for serum PCR and blood culture for the 
Table 3 Relationship between presence of invasive candidiasis and patient risk factors

\begin{tabular}{|c|c|c|c|c|c|c|c|c|c|c|c|c|c|c|c|}
\hline & \multicolumn{5}{|c|}{ Candida score $\geq 3$} & \multicolumn{5}{|c|}{ Sepsis with $\geq 3$ risk factors } & \multicolumn{5}{|c|}{$\begin{array}{l}\text { Sepsis in patients with } \\
\text { immunosuppression }\end{array}$} \\
\hline & \multicolumn{2}{|l|}{ Yes } & \multicolumn{2}{|c|}{ No } & \multirow[t]{2}{*}{$P^{\mathrm{a}}$} & \multicolumn{2}{|l|}{ Yes } & \multicolumn{2}{|l|}{ No } & \multirow[t]{2}{*}{$P^{\mathbf{a}}$} & \multicolumn{2}{|c|}{$\overline{\text { Yes }}$} & \multicolumn{2}{|l|}{ No } & \multirow[t]{2}{*}{$P^{\mathbf{a}}$} \\
\hline & $n$ & $\%$ & $n$ & $\%$ & & $n$ & $\%$ & $n$ & $\%$ & & $n$ & $\%$ & $n$ & $\%$ & \\
\hline \multicolumn{16}{|c|}{ Serum PCR } \\
\hline Negative & 119 & 90.8 & 40 & 90.9 & 0.989 & 147 & 90.7 & 12 & 92.3 & $>0.05$ & 35 & 97.2 & 124 & 89.2 & $>0.05$ \\
\hline Positive & 12 & 9.2 & 4 & 9.1 & & 15 & 9.3 & 1 & 7.7 & & 1 & 2.8 & 15 & 10.8 & \\
\hline \multicolumn{16}{|c|}{ Blood culture } \\
\hline Negative & 118 & 90.1 & 43 & 97.7 & 0.106 & 149 & 92.0 & 12 & 92.3 & $>0.05$ & 33 & 91.7 & 128 & 92.1 & $>0.05$ \\
\hline Positive & 13 & 9.9 & 1 & 2.3 & & 13 & 8.0 & 1 & 7.7 & & 3 & 8.3 & 11 & 7.9 & \\
\hline \multicolumn{16}{|c|}{ Abdominal fluid } \\
\hline Negative & 101 & 77.1 & 42 & 95.5 & 0.006 & 131 & 80.9 & 12 & 92.3 & $>0.05$ & 33 & 91.7 & 110 & 79.1 & $>0.05$ \\
\hline Positive & 30 & 22.9 & 2 & 4.5 & & 31 & 19.1 & 1 & 7.7 & & 3 & 8.3 & 29 & 20.9 & \\
\hline \multicolumn{16}{|c|}{$\begin{array}{l}\text { Blood } \\
\text { culture }+ \text { abdominal } \\
\text { fluid }\end{array}$} \\
\hline Negative & 96 & 73.3 & 41 & 93.2 & 0.006 & 125 & 77.2 & 12 & 92.3 & $>0.05$ & 32 & 88.9 & 105 & 75.5 & $>0.05$ \\
\hline Positive & 35 & 26.7 & 3 & 6.8 & & 37 & 22.8 & 1 & 7.7 & & 4 & 11.1 & 34 & 24.5 & \\
\hline
\end{tabular}

PCR polymerase chain reaction

${ }^{a}$ Chi-squared test

detection of Candida spp. in patients admitted to ICUs in Spain and in whom empirical antifungal treatment was initiated because of suspected invasive candidiasis. Although a metaanalysis of studies evaluating PCR diagnosis for invasive candidiasis was published in 2011 [36], the patient populations assessed in this study were highly heterogeneous in terms of country of origin, age, underlying conditions, etc. In contrast, the current study provides information on a specific group of patients from Spain. It is important determine the issues that lead physicians to initiate antifungal therapy [39] and to perform PCR simultaneously with blood culture to avoid storage problems.

In this study, the rates of Candida detection in blood samples were low, whether using serum PCR $(16 / 175,9.1 \%)$ or blood culture (14/ $175,8.0 \%)$. Candida spp. appeared to be more readily detected in the abdominal fluid (32/49,
65.3\%). As could be expected, C. albicans was the most frequently detected species, although C. glabrata, C. krusei, C. tropicalis and C. parapsilosis were also identified. These five species represent the most common Candida pathogens $[20,30]$.

The sensitivity of serum PCR relative to blood culture for the detection of Candida spp. was $21.4 \%$. While the results for PCR relative to abdominal fluid were marginally higher $(31.3 \%)$, the sensitivity of serum PCR relative to the composite measure (Candida positivity in blood culture or abdominal fluid) decreased even further (15.8\%) (Table 2).

While the specificity and NPV values for PCR obtained in the current study were similar to those previously reported in other studies $[25,36,40]$, the sensitivity and PPV values achieved (Table 2) were much lower overall. This may be due to the use of serum samples for 
Table 4 Logistic regression models for risk factors

\begin{tabular}{lllll}
\hline Risk factor & Effect & OR & $\mathbf{9 5 \%}$ CI & $\boldsymbol{P}$ \\
\hline $\begin{array}{l}\text { Candida infection according to blood culture } \\
\text { Candida score } \geq 3\end{array}$ & Yes vs. no & 7.00 & $(0.69-71.31)$ & 0.100 \\
Sepsis with $\geq 3$ risk factors & Yes vs. no & 0.51 & $(0.04-5.93)$ & 0.594 \\
Sepsis in patients with immunosuppression & Yes vs. no & 1.62 & $(0.37-7.03)$ & 0.521 \\
Candida infection according to serum PCR & & & & \\
Candida score $\geq 3$ & Yes vs. no & 0.71 & $(0.20-2.49)$ & 0.588 \\
Sepsis with $\geq 3$ risk factors & Yes vs. no & 0.39 & $(0.03-5.26)$ & 0.481 \\
Sepsis in patients with immunosuppression & Yes vs. no & 0.14 & $(0.01-1.77)$ & 0.128 \\
Candida infection according to blood culture + abdominal fluid & & & \\
Candida score $\geq 3$ & Yes vs. no & 4.15 & $(1.15-14.98)$ & 0.030 \\
Sepsis with $\geq 3$ risk factors & Yes vs. no & 1.36 & $(0.14-12.93)$ & 0.789 \\
Sepsis in patients with immunosuppression & Yes vs. no & 0.62 & $(0.19-2.05)$ & 0.432 \\
\hline
\end{tabular}

$C I$ confidence interval, $O R$ odds ratio, $P C R$ polymerase chain reaction

Table 5 Risk factors associated with the presence of invasive candidiasis according to blood culture (adjusted binary logistic regression model with a bilateral significance level of 0.10 , Chi-squared test)

\begin{tabular}{lllll}
\hline Risk factor & Effect & OR & 95\% CI & $\boldsymbol{P}$ \\
\hline Candida infection according to blood culture & & & & \\
Abdominal surgery & Yes vs. no & 4.342 & $(0.898-20.998)$ & 0.068 \\
Admission to ICU & $>15$ days vs. $\leq 15$ days & 2.560 & $(0.793-8.263)$ & 0.090 \\
Sepsis to patients with some immunosuppression criterion & Yes vs. no & 0.556 & $(0.134-2.308)$ & 0.419 \\
\hline
\end{tabular}

$C I$ confidence interval, $I C U$ intensive care unit, $O R$ odds ratio

our PCR assays rather than whole blood, according to each site's usual practice; greater sensitivity has been reported when PCR is performed in whole-blood samples [36; the CANDINEO Study [40].

The results from our analyses showed a very low concordance rate between the results of serum PCR and blood culture (kappa index: 0.125). The degree of concordance was not enhanced by using the composite measure, since the kappa index for serum PCR versus the composite measure was 0.107 , again indicating a very low degree of concordance between serum PCR and the other diagnostic techniques used in this study.
Logistic regression analyses of the risk factors that, in clinical terms, may be considered to contribute to the probability of developing a Candida infection indicated a direct relationship between a Candida score $>3$ and a higher probability of invasive candidiasis (as diagnosed using blood culture or the composite measure). None of the other risk factors showed a particular association with the risk of developing invasive candidiasis.

An additional binary logistic regression model was used to determine which factors are most related to the presence of candidiasis, according to the blood culture result. Abdominal surgery and a longer ICU stay ( $>15$ days) 
showed a positive trend towards being risk factors for the presence of Candida spp. in blood cultures. These findings are consistent with previous studies, which have also found that abdominal surgery and length of stay at the ICU are associated with higher risk of invasive candidiasis [1, 41]. Conversely, we cannot explain why a very low concordance was found between blood culture and PCR; it may be necessary to perform PCR at the same time as blood culture.

The apparent lack of relationship between risk factors and the presence of invasive candidiasis using PCR is somewhat surprising, especially when this technique has proved to be very sensitive in similar ICU settings [25]. However, in another study the diagnostic accuracy of PCR was also low because the tests were performed in frozen samples [40]; this factor may be applicable to our study because of the freezing of samples prior to dispatch to the Reference Centre. In addition, there was no significant relationship between invasive candidiasis (as assessed using blood culture) and a number of known risk factors. This may also be attributable to the low sensitivity of blood cultures to diagnose Candida infections and the broad definition of a 'high-risk' population. Patients with just one of three positive blood cultures might have shown a negative PCR because of a low burden of Candida spp. Likewise, regarding the origin of candidaemia, abdominal fluid from a peritoneal catheter may present a higher Candida burden than an intraabdominal infection and therefore diminish the sensitivity of PCR. Difficulties with distinguishing Candida colonisation from intra-abdominal invasive candidiasis may contribute to the low sensitivity of PCR in some patients [30].

Due to the non-interventional nature of this study, all patient examinations and follow-up visits were performed in line with the investigators' usual clinical practice. The low cure rate is of note, but it is not possible to speculate on the reasons for this as information relating to treatment strategies is not available. The lack of use of follow-up PCR is also notable, and the possible reasons for this may include discharge of patients to other units and therefore loss to follow-up or improvement of their clinical condition and therefore no additional samples or investigations were needed.

In a high proportion of cases, a follow-up blood culture was not conducted, i.e. the physicians in this study appeared to rely on signs and symptoms to determine whether the Candida infection had resolved. Other recent studies that have evaluated PCR as a diagnostic tool for invasive candidiasis have reported conflicting results $[25,40,42]$. In a Spanish study amongst critically ill patients admitted to the ICU, PCR appeared to have a high sensitivity (96.3\%), specificity (97.3\%) and strong positive predictive value $(92.8 \%)$, especially in patients with deep-seated disease (sensitivity $90.9 \%$ versus $45.4 \%$ for blood culture) [25], and therefore the authors concluded that PCR may be a useful test for confirming a diagnosis of invasive candidiasis [25]. However, in our study of high-risk patients, the incidence of candidaemia was strikingly lower than in the previous study [25]. Similarly, in another recent Spanish study, PCR had a low discriminating capacity (sensitivity $84.00 \%$; PPV $26.9 \%$ ), whether used alone or in combination with other antibody-based diagnostic tests [40]. In the study by Nguyen et al. [42], Candida PCR testing was more sensitive than blood cultures among patients with deep-seated candidiasis ( $88 \%$ versus $17 \%$, respectively), and it significantly enhanced the ability of blood culture to detect invasive candidiasis by increasing overall sensitivity to $98 \%$ when used in combination with blood culture [42].

Nonetheless, significant shortcomings for PCR testing still remain, for instance, a relatively low sensitivity because of the low quantity of Candida cells in the blood [1], sample susceptibility to contamination [25] and other issues that need to be overcome before it can be used routinely and effectively in clinical practice (e.g. the lack of a standardised assay) [40]. Also, the lack of commercially available methods that are easy-to-use is still limiting [1]; different methodologies between centres performing these assays may also affect the consistency of clinical data which are generated.

Another limitation of the study was that information on patients who failed screening 
was not collected; for this reason, the possibility of selection bias cannot be excluded. Due to its observational nature, additional limitations of this study may have included variations in physicians' clinical practice among all 27 participating centres. In addition, a second PCR that might provide information as to whether the initial negative results were truly negative was performed in very few patients. Furthermore, a number of pre-specified secondary end points (such as the prognostic value and diagnostic sensitivity of serum PCR in invasive candidiasis) were not assessed as planned because of low patient numbers or a low prevalence of invasive candidiasis in our samples. It would also be interesting to evaluate whether the outcomes of patients diagnosed by PCR and blood culture differ, but this was beyond the scope of the current study.

\section{CONCLUSIONS}

In this observational, prospective, multicentre study amongst critically ill patients at a high risk of invasive candidiasis who had been admitted to an ICU, there was a low rate of concordance between serum PCR and blood culture, and serum PCR and culture of abdominal fluid samples, for the diagnosis of Candida infections. Thus, the PCR methodology used in the current study would not provide a clear diagnostic advantage in these patients. Within this patient population, there was a direct and statistically significant association between a Candida score $>3$ and a higher probability of invasive candidiasis. Other evidence has substantiated the need for rapid and accurate methods to detect Candida spp., but this study highlights the importance of refining and validating such tests as well as establishing the risk factors for identification of critically ill patients who require early therapeutic intervention.

\section{ACKNOWLEDGEMENTS}

Funding. This study (conception and design; data acquisition, analysis and interpretation) and the journal's article processing charges were funded by Astellas Pharma Inc. All authors had full access to all of the data in this study and take complete responsibility for the integrity of the data and accuracy of the data analysis.

Medical Writing Assistance. Medical writing support, which was funded by Astellas Pharma Inc., was provided by David Griffiths PhD (on behalf of Bioscript Medical).

Authorship. All named authors meet the International Committee of Medical Journal Editors (ICMJE) criteria for authorship for this article, take responsibility for the integrity of the work as a whole, and have given their approval for this version to be published.

Authorship Contribution. All authors contributed to the study design and methodology, screening and inclusion of patients, sample collection, follow-up assessments, data analysis and interpretation, critical review of the manuscript with important contributions for scientific content, and approval of the final draft.

List of Investigators. The authors would like to thank the participants of this study and other members of the MICAFEM Study Group (Dr. Luis Tamayo, Dr. Carmen Ramos, Dr. Jose Luis Teja, Dr. M. Pia Roiz, Dr. Felipe Bobillo, Dr. Miguel A. Bratos, Dr. M. Carmen de la Torre, Dr. Goretti Saucas, Dr. Juan Carlos Ballesteros, Dr. Inmaculada García, Dr. Paula Vera, Dr. Ferrando Sánchez, Dr. José Peral, Dr. Emilio Bouza, Dr. Patricia Muñoz, Dr. M. Victoria de la Torre, Dr. M. Victoria García, Dr. Pamela Saénz, Dr. Carme Martí, Dr. Carmen J. Fernández, Dr. Patricia Ordoñez, Dr. Iñaki Catalán, Dr. M. Dolors Estivill, Dr. Isidro Prieto, Dr. Elia Gómez, Dr. Ricardo Serrano, Dr. Joaquín Plazas, Dr. José Castaño, Dr. M. Dolores Pérez) who enrolled patients into the study. The authors would also like to thank Dr. Miguel A. Romera, Dr. Beatriz Orden, Dr. Andrés Carretero, Dr. Mariano Villaseñor, Dr. Elena Escudero, Dr. Miguel A. Blasco, Dr. Soledad Cúetara, Dr. Paula Ramírez, Dr. Javier Pemán, Dr. Rafael Zaragoza, Dr. 
Fátima Martín, Dr. Luis M. Ruiz, Dr. Pilar Luque and Dr. M. Soledad Salvo as well as the investigators who are members of Grupo de Estudio de Infecciones en el Paciente Crítico de la Sociedad Española de Enfermedades Infecciosas y Microbiología Clínica (GEIPC-SEMICYUC) and Grupo de Trabajo de Enfermedades Infecciosas y Sepsis (GTEIS), for their participation in the study.

Disclosures. Mercedes Nieto has received financial support from Astellas Pharma Inc. and MSD (consultancy and travel fees) as well as from Pfizer and Novartis (travel fees). Ricard Ferrer has received financial support from Grifols and MSD (consultancy fees). Paloma Merino has received a research grant and consultancy fees from Astellas Pharma Inc. Paloma Anguita is an employee of Astellas Pharma Inc. Juan Carlos Robles, Manuel Causse, Leticia Gutiérrez, Maria Cruz Perez, Mariona Xercavins, Eugenio Herrero, Elia Sirvent and Cristina Fernández have nothing to disclose.

Compliance with Ethics Guidelines. An Independent Ethics Committee or an Institutional Review Board reviewed and approved the protocol, protocol amendments and patient information before the start of the study. The "master" ethics committee was the H. Clinico San Carlos committee (a list of all ethics approval bodies that approved the study in the various centres is included in the supplementary material). The study was conducted in accordance with the principles of the 1964 Declaration of Helsinki and its later amendments, Good Clinical Practice guidelines and International Conference on Harmonisation guidelines. All patients provided written informed consent to participate before the start of the study.

Data Availability. All relevant data are included in this manuscript. The protocol and complete data sets generated and/or analysed during the current study are not publicly available, but are available from the corresponding author on reasonable request. Access to anonymized individual participant level data will not be provided for this trial as it meets one or more of the exceptions described on http://www. clinicalstudydatarequest.com under "Sponsor Specific Details for Astellas".

Open Access. This article is distributed under the terms of the Creative Commons Attribution-NonCommercial 4.0 International License (http://creativecommons.org/licenses/ by-nc/4.0/), which permits any noncommercial use, distribution, and reproduction in any medium, provided you give appropriate credit to the original author(s) and the source, provide a link to the Creative Commons license, and indicate if changes were made.

\section{REFERENCES}

1. Eggimann P, Bille J, Marchetti O. Diagnosis of invasive candidiasis in the ICU. Ann Intensive Care. $2011 ; 1: 37$.

2. Azie N, Neofytos D, Pfaller M, Meier-Kriesche HU, Quan SP, Horn D. The PATH (Prospective Antifungal Therapy) Alliance(R) registry and invasive fungal infections: update 2012. Diagn Microbiol Infect Dis. 2012;73:293-300.

3. Guinea J. Global trends in the distribution of Candida species causing candidemia. Clin Microbiol Infect. 2014;20(Suppl 6):5-10.

4. Montagna MT, Caggiano G, Lovero G, et al. Epidemiology of invasive fungal infections in the intensive care unit: results of a multicenter Italian survey (AURORA Project). Infection. 2013;41:645-53.

5. Kullberg BJ, Arendrup MC. Invasive candidiasis. N Engl J Med. 2015;373:1445-56.

6. Arendrup MC. Candida and candidaemia. Susceptibility and epidemiology. Dan Med J. 2013;60:B4698.

7. Horn DL, Neofytos D, Anaissie EJ, et al. Epidemiology and outcomes of candidemia in 2019 patients: data from the prospective antifungal therapy alliance registry. Clin Infect Dis. 2009;48:1695-703.

8. Kett DH, Azoulay E, Echeverria PM, Vincent JL, Extended Prevalence of Infection in ICU Study (EPIC II) Group of Investigators. Candida bloodstream infections in intensive care units: analysis of 
the extended prevalence of infection in intensive care unit study. Crit Care Med. 2011;39:665-70.

9. Ruhnke M. Antifungal stewardship in invasive Candida infections. Clin Microbiol Infect. 2014;20(Suppl 6):11-8.

10. Gagne JJ, Goldfarb NI. Candidemia in the in-patient setting: treatment options and economics. Expert Opin Pharmacother. 2007;8:1643-50.

11. Morgan J, Meltzer MI, Plikaytis BD, et al. Excess mortality, hospital stay, and cost due to candidemia: a case-control study using data from population-based candidemia surveillance. Infect Control Hosp Epidemiol. 2005;26:540-7.

12. Rentz AM, Halpern MT, Bowden R. The impact of candidemia on length of hospital stay, outcome, and overall cost of illness. Clin Infect Dis. 1998;27:781-8.

13. Pfaller MA, Diekema DJ. Epidemiology of invasive candidiasis: a persistent public health problem. Clin Microbiol Rev. 2007;20:133-63.

14. Yang SP, Chen YY, Hsu HS, Wang FD, Chen LY, Fung CP. A risk factor analysis of healthcare-associated fungal infections in an intensive care unit: a retrospective cohort study. BMC Infect Dis. 2013;13:10.

15. Holley A, Dulhunty J, Blot S, et al. Temporal trends, risk factors and outcomes in albicans and non-albicans candidaemia: an international epidemiological study in four multidisciplinary intensive care units. Int J Antimicrob Agents. 2009;33(554):e1-7.

16. Jorda-Marcos R, Alvarez-Lerma F, Jurado M, et al. Risk factors for candidaemia in critically ill patients: a prospective surveillance study. Mycoses. 2007;50:302-10.

17. Leon C, Ruiz-Santana S, Saavedra P, et al. A bedside scoring system ("Candida score") for early antifungal treatment in nonneutropenic critically ill patients with Candida colonization. Crit Care Med. 2006;34:730-7.

18. Leon C, Ruiz-Santana S, Saavedra P, et al. Usefulness of the "Candida score" for discriminating between Candida colonization and invasive candidiasis in non-neutropenic critically ill patients: a prospective multicenter study. Crit Care Med. 2009;37:1624-33.

19. Pittet D, Monod M, Suter PM, Frenk E, Auckenthaler R. Candida colonization and subsequent infections in critically ill surgical patients. Ann Surg. 1994;220:751-8.
20. Calandra T, Roberts JA, Antonelli M, Bassetti M, Vincent JL. Diagnosis and management of invasive candidiasis in the ICU: an updated approach to an old enemy. Crit Care. 2016;20:125.

21. Skrobik Y, Laverdiere M. Why Candida sepsis should matter to ICU physicians. Crit Care Clin. 2013;29:853-64.

22. Leroy O, Gangneux JP, Montravers P, et al. Epidemiology, management, and risk factors for death of invasive Candida infections in critical care: a multicenter, prospective, observational study in France (2005-2006). Crit Care Med. 2009;37:1612-8.

23. Wisplinghoff $H$, Bischoff T, Tallent SM, Seifert $H$, Wenzel RP, Edmond MB. Nosocomial bloodstream infections in US hospitals: analysis of 24,179 cases from a prospective nationwide surveillance study. Clin Infect Dis. 2004;39:309-17.

24. Shorr AF, Gupta V, Sun X, Johannes RS, Spalding J, Tabak YP. Burden of early-onset candidemia: analysis of culture-positive bloodstream infections from a large U.S. database. Crit Care Med. 2009;37:2519-26 (quiz 35).

25. Fortun J, Meije Y, Buitrago MJ, et al. Clinical validation of a multiplex real-time PCR assay for detection of invasive candidiasis in intensive care unit patients. J Antimicrob Chemother. 2014;69:3134-41.

26. Olaechea-Astigarraga PM, Alvarez-Lerma F, Palomar-Martinez $\mathrm{M}$, et al. Trends in systemic antifungal use in critically ill patients. Multicenter observational study, 2006-2010. Enferm Infecc Microbiol Clin. 2012;30:435-40.

27. Azoulay E, Dupont H, Tabah A, et al. Systemic antifungal therapy in critically ill patients without invasive fungal infection. Crit Care Med. 2012;40:813-22.

28. Cornely OA, Bassetti M, Calandra T, et al. ESCMID* guideline for the diagnosis and management of Candida diseases 2012: non-neutropenic adult patients. Clin Microbiol Infect. 2012;18(Suppl 7):19-37.

29. McDonald LC, Gerding DN, Johnson S, et al. Clinical practice guidelines for clostridium difficile infection in adults and children: 2017 update by the Infectious Diseases Society of America (IDSA) and Society for Healthcare Epidemiology of America (SHEA). Clin Infect Dis. 2018;66:987-94.

30. Pappas P, Kauffman C, Andes D, et al. Clinical practice guideline for the management of candidiasis: 2016 update by the Infectious Diseases Society of America. Clin Infect Dis. 2016;62:e1-50. 
31. Shields RK, Nguyen MH, Clancy CJ. Clinical perspectives on echinocandin resistance among Candida species. Curr Opin Infect Dis. 2015;28:514-22.

32. Munoz P, Valerio M, Vena A, Bouza E. Antifungal stewardship in daily practice and health economic implications. Mycoses. 2015;58(Suppl 2):14-25.

33. Garey KW, Rege M, Pai MP, et al. Time to initiation of fluconazole therapy impacts mortality in patients with candidemia: a multi-institutional study. Clin Infect Dis. 2006;43:25-31.

34. Morrell M, Fraser VJ, Kollef MH. Delaying the empiric treatment of candida bloodstream infection until positive blood culture results are obtained: a potential risk factor for hospital mortality. Antimicrob Agents Chemother. 2005;49:3640-5.

35. Clancy CJ, Nguyen MH. Finding the "missing 50\%" of invasive candidiasis: how nonculture diagnostics will improve understanding of disease spectrum and transform patient care. Clin Infect Dis. 2013;56:1284-92.

36. Avni T, Leibovici L, Paul M. PCR diagnosis of invasive candidiasis: systematic review and metaanalysis. J Clin Microbiol. 2011;49:665-70.
37. Peman J, Zaragoza R. Combined use of nonculturebased lab techniques in the diagnosis and management of critically ill patients with invasive fungal infections. Expert Rev Anti Infect Ther. 2012;10:1321-30.

38. McHugh ML. Interrater reliability: the kappa statistic. Biochem Med (Zagreb). 2012;22:276-82.

39. Ramos JT, Villar S, Bouza E, et al. Performance of a quantitative PCR-based assay and beta-d-glucan detection for diagnosis of invasive candidiasis in very-low-birth-weight preterm neonatal patients (CANDINEO Study). J Clin Microbiol. 2017;55:2752-64.

40. Leon C, Ruiz-Santana S, Saavedra P, et al. Contribution of Candida biomarkers and DNA detection for the diagnosis of invasive candidiasis in ICU patients with severe abdominal conditions. Crit Care. 2016;20:149.

41. Lagunes L, Rello J. Invasive candidiasis: from mycobiome to infection, therapy, and prevention. Eur J Clin Microbiol Infect Dis. 2016;35:1221-6.

42. Nguyen MH, Wissel MC, Shields RK, et al. Performance of Candida real-time polymerase chain reaction, beta-D-glucan assay, and blood cultures in the diagnosis of invasive candidiasis. Clin Infect Dis. 2012;54:1240-8. 